\title{
ANÁLISE DE COMPOSTOS FENÓLICOS TOTAIS E ATIVIDADE ANTIOXIDANTE DE CASCA E POLPA DE MAÇÃ E SUAS RESPECTIVAS FARINHAS
}

Analysis of total phenolic compounds and antioxidant activity of apple peel and pulp and their respective flours

Análisis de compuestos fenólicos totales y actividad antioxidante de cáscara y pulpa de manzana y sus respectivas harinas

Daphynni Carolinne Moreira Morais*1, Danilo José Machado de Abreu², Dianiny de Cassia Sousa Mendes², Ulisses Rodrigues de Alencar ${ }^{2}$, Katiúcia Alves Amorim², Clarissa Damiani $^{2}$

${ }^{1}$ Programa de Pós-graduação em Ciência e Tecnologia de Alimentos (PPGCTA), Universidade Federal do Tocantins (UFT), Palmas, Brasil.

${ }^{2}$ Programa de Pós-graduação em Ciência e Tecnologia de Alimentos, Universidade Federal do Goiás (UFG), Goiânia, Brasil.

*Correspondência: Laboratório de Ciências, Instituto de Ensino Superior, Av. NS 15, 109 Norte, Palmas, Tocantins, Brasil. CEP:77.010-090.e-mail artigo@artigo.com.

\section{Artigo recebido em 29/04/2019 aprovado em 03/05/2019 publicado em 16/06/2019.}

\section{RESUMO}

A maçã é um dos frutos mais apreciados no mundo e consolidado mundialmente. De acordo com a Associação Brasileira de Produtores de maçã (ABPM), a produção brasileira concentra-se, principalmente, nos estados do Rio Grande do Sul, Santa Catarina e Paraná, correspondendo a 99\% da produção total. Dos frutos produzidos, grande parte são consumidos in natura e na produção de sucos, gerando resíduos agroindustriais. O estudo teve por objetivo quantificar o teor de compostos fenólicos e potencial antioxidante de casca e polpa de maçã in natura e de suas farinhas. Os resultados do teor de fenólicos mostraram que tanto a farinha da casca (1751,38mgGAE/100g) como a farinha da polpa $(308,52 \mathrm{mgGAE} / 100 \mathrm{~g})$ apresentaram diferenças significativas em relação ao fruto in natura. Pelo método DPPH (IC50), apresentaram-se menores valores as amostras de farinhas de casca e da polpa de maçã em relação a in natura, porém, ambas as amostras avaliadas, obtiveram atividade antioxidante, mostrando a viabilidade do aproveitamento como ingrediente enriquecedor.

Palavras-chave: Maçã, Compostos fenólicos, Atividade Antioxidante.

\section{ABSTRACT}

The apple is one of the most appreciated fruits in the world and consolidated worldwide. According to the Brazilian Association of Apple Producers (ABPM), Brazilian production is mainly concentrated in the states of Rio Grande do Sul, Santa Catarina and Paraná, corresponding to 99\% of the total production. Of the fruits produced, most are consumed in natura and in the production of juices, generating agroindustrial residues. The objective of this study was to quantify the content of phenolic compounds and the antioxidant potential of peel and apple pulp in natura and their flours. The results of the phenolic content showed that both the bark meal (1751,38mgGAE/100g) and the pulp flour (308,52mgGAE / 100g) presented significant differences in relation to the fruit in natura. By the DPPH method (IC 50), the values of the shell and apple pulp samples were lower in relation to in natura, but both evaluated samples obtained antioxidant activity, showing the viability of the use as an enriching ingredient.

Keywords: Apple, Phenolic compounds, Antioxidant activity.

\section{RESUMEN}


La manzana es uno de los frutos más apreciados en el mundo y consolidado mundialmente. De acuerdo con la Asociación Brasileña de Productores de manzana (ABPM), la producción brasileña se concentra principalmente en los estados de Rio Grande do Sul, Santa Catarina y Paraná, correspondiendo al 99\% de la producción total. De los frutos producidos, gran parte se consumen in natura y en la producción de jugos generando residuos agroindustriales. El estudio tuvo por objetivo cuantificar el contenido de compuestos fenólicos y potencial antioxidante de cáscara y pulpa de manzana in natura y de sus harinas. Los resultados del contenido de fenólicos mostraron que tanto la harina de la cáscara (1751,38 mgGAE/100g) como la harina de la pulpa (308,52mgGAE/100g) presentaron diferencias significativas en relación al fruto in natura. Por el método DPPH (IC50), se presentaron menores valores las muestras de harinas de cáscara y de la pulpa de manzana em relación a la in natura, pero ambas muestras evaluadas, obtuvieron actividad antioxidante, mostrando la viabilidade del aprovechamiento como ingrediente enriquecedor.

Descriptores: Manzana, Compuestos fenólicos.

\section{INTRODUÇÃO}

A maçã é um fruto apreciado mundialmente, onde seu maior consumo é na forma in natura, apresentando altos valores de vitaminas do complexo $\mathrm{B}$, vitaminas $\mathrm{C}$ e $\mathrm{E}$, minerais como potássio, além de possuir fibras, fornecendo, principalmente, $10 \%$ da ingestão diária recomendada desses nutrientes (SANTOS, 2017).

Possui propriedades benéficas que desempenham papel fundamental na manutenção da saúde humana, no qual atua na prevenção e controle de doenças cardiovasculares, pulmonares, diabetes mellitus tipo 2, alergias, obesidade, colesterolemia, atividade carcinogênica, dentre outros (BOYER E LIU, 2004; SHAHIDI,2012).

De acordo com Tsau et al. (2005), a maçã apresenta elevados teores de fitonutrientes, sendo flavonoides, polifenóis e ácidos fenólicos, encontrados na polpa e em maior quantidade na casca, fornecendo como benefícios os antioxidantes. Os compostos fenólicos podem, também, contribuir para as características sensoriais do fruto como a cor, sabor e produção de aromas. (QUEIJI ET AL.,2010)

Dessa maneira, o objetivo desse estudo foi quantificar o teor de compostos fenólicos e avaliar a atividade antioxidante da maçã gala, avaliando a sua casca, polpa e suas respectivas farinhas.

\section{MATERIAIS E MÉTODOS}

\section{Matéria prima}

Os frutos foram adquiridos no comércio local de Goiânia - GO, onde o critério foi o estádio de maturidade, sendo escolhidas maçãs maduras com coloração vermelha. Foram encaminhados para a Universidade Federal do Goiás, no laboratório Análise de Alimentos. Foram lavados com água corrente e sanitizados com solução de hipoclorito de sódio a 200 ppm por um tempo de 15 minutos. Após higienizadas, realizou-se a separação manual de casca e polpa para as análises in natura e, posteriormente, estas foram submetidas a secagem em estufa, de circulação de ar forçado, a $60^{\circ} \mathrm{C}$, por 24 horas para casca e 72 horas a polpa de maçã para a obtenção das farinhas que foram armazenadas em freezer a $-4^{\circ} \mathrm{C}$.

\section{Preparo dos extratos}

Os extratos hidroalcoólicos, utilizados nas análises de compostos fenólicos totais e atividade antioxidante por DPPH foram preparados ao abrigo da luz, nos quais foram pesados 2,5 g de amostra com 50 $\mathrm{mL}$ de álcool 70, macerados, filtrados com auxílio de papel filtro e armazenados em vidro âmbar em freezer a $-4^{\circ} \mathrm{C}$.

Compostos fenólicos totais: A quantificação de compostos fenólicos totais, foi realizada utilizando-se o reagente de Folin-Ciocalteau, conforme metodologia descrita por Waterhouse (2002), com leitura em 
espectrofotômetro digital (Rayleigh, UV-1800), a 750 nm. Baseou-se no estabelecimento de uma curva padrão de ácido gálico, na faixa de 5 a $50 \mathrm{mg}$ L-1. Os resultados foram expressos em $\mathrm{mg}$ de equivalentes de ácido gálico (GAE) por $100 \mathrm{~g}$ de amostra.

Determinação da capacidade de sequestrar radicais livres (DPPH): Foi utilizada a metodologia de Brand-Willians et al. (1995) com modificações. Para a análise das amostras, adicionou-se a 3,9 mL da solução metanólica de DPPH e uma alíquota de $0,1 \mathrm{~mL}$ do extrato. As leituras foram realizadas em espectrofotômetro a $517 \mathrm{~nm}$, até a estabilização da absorbância. Todas as determinações foram acompanhadas de um controle. A queda na leitura da densidade ótica das amostras foi comparada com o controle, estabelecendo-se a porcentagem de descoloração do radical DPPH, conforme a Equação 1:

Ativ. Antiox. $(\%)=($ Abscontrole - Absamostra $) x$ 100/ Abscontrole (1)

Após encontrar a \% de descoloração, foi calculada a quantidade necessária de amostra para descolorir $50 \%$ do DPPH, os resultados foram expressos em IC50 (mg/ml).

\section{Análise estatística}

Todas as análises foram realizadas em triplicata e os resultados expressos em média e desvio padrão. Os resultados foram submetidos à análise de variância, seguido do teste $\mathrm{T}$ a $5 \%$ de probabilidade com o auxílio do programa SISVAR 5.6 (Ferreira, 2010).

\section{RESULTADOS E DISCUSSÃO}

Os resultados das análises de compostos fenólicos e atividade antioxidante da casca da maçã in natura e respectiva farinha estão apresentados na
Tabela 1 e os resultados das análises da polpa de maçã in natura e respectiva farinha na Tabela 2.

Quando comparados os resultados das amostras in natura com suas respectivas farinhas, observou-se que estas diferiram significativamente entre si pelo teste $T(p<0,05)$.

Tabela 1. Compostos fenólicos e atividade antioxidante da casca in natura e farinha da casca da maçã gala, oriunda do comércio local de Goiás- Brasil.

\begin{tabular}{ccc}
\hline & CM & FCM \\
\hline $\begin{array}{c}\text { Fenólicos } \\
\text { totais } \\
(\mathbf{m g G A E} / 100 g)\end{array}$ & $212,12 \pm 1,44 \mathrm{a}$ & $1751,38 \pm 0,58 \mathrm{~b}$ \\
\hline $\begin{array}{c}\text { DPPH } \\
\text { (IC 50\%) }\end{array}$ & $5,33 \pm 0,36 \mathrm{a}$ & $2,86 \pm 0,06 \mathrm{~b}$ \\
\hline
\end{tabular}

Valores expressos correspondem às médias \pm desvio padrão de três repetições. Letras iguais na mesma linha não apresentam diferença estatística entre si (Teste T, p <0,05). $\mathrm{CM}=$ Casca de maçã. FCMA = Farinha de casca de maçã. GAE = ácido gálico. IC 50 - concentração de extrato $(\mathrm{mg} / \mathrm{mL})$ capaz de reduzir 50\% do DPPH inicial.

Tabela 2. Compostos fenólicos e atividade antioxidante da polpa in natura e farinha da polpa da maçã gala, oriunda do comércio local de Goiás- Brasil.

\begin{tabular}{ccc}
\hline & PM & FPM \\
\hline $\begin{array}{c}\text { Fenólicos } \\
\text { totais } \\
(\mathbf{m g G A E} / 100 g)\end{array}$ & $36,69 \pm 0,80 \mathrm{a}$ & $308,52 \pm 0,74 \mathrm{~b}$ \\
\hline $\begin{array}{c}\text { DPPH } \\
\text { (IC 50\%) }\end{array}$ & $22,45 \pm 0,05 \mathrm{a}$ & $3,82 \pm 0,07 \mathrm{~b}$ \\
\hline
\end{tabular}

Valores expressos correspondem às médias \pm desvio padrão de três repetições. Letras iguais na mesma linha não apresentam diferença estatística entre si (Teste $T, p<0,05$ ). $\mathrm{PM}=$ Polpa de maçã. FPM = Farinha da polpa da maçã. GAE = ácido gálico. IC 50 - concentração de extrato $(\mathrm{mg} / \mathrm{mL})$ capaz de reduzir 50\% do DPPH inicial.

De acordo com a tabela 01 e a tabela 02 , os compostos fenólicos da farinha da casca de maçã e da farinha da polpa da maçã apresentaram elevados teores e como tem sido mostrado no trabalho de Ito (2015), estão entre os fitoquímicos que podem ajudar a prevenir vários tipos de câncer e doenças cardiovasculares, devido aos seus efeitos via ação antioxidante de forma a minimizar o estresse oxidativo. 
Soares (2008) mostrou em seu trabalho que a maçã apresenta elevado teor de compostos fenólicos, encontrados na polpa e na casca. Conforme os dados obtidos, a quantificação de compostos fenólicos na maçã, em forma de farinha, apresentou maiores quantidades, tanto na casca como da polpa da fruta, potencializando a industrialização da farinha como alternativa de aproveitamento (NERIS et al.2018).

De acordo com Alberti (2014), quanto menor o valor de (IC 50) maior o potencial antioxidante, constatando maior eficiência na farinha da casca de maçã $(2,86 \mathrm{mg} / \mathrm{mL})$ em relação a casca de maçã in natura $(5,33 \mathrm{mg} / \mathrm{mL})$. Entre a polpa da maçã

\section{CONCLUSÃO}

O teor de compostos fenólicos e atividade antioxidante variaram entre as amostras de casca de maçã x farinha da casca de maçã e de polpa de maçã $x$ farinha da polpa de maçã, indicando que o simples processamento desses resíduos (cascas) em forma de farinhas, apresentou influência na composição fenólica, ressaltando a possibilidade de sua utilização em diversas preparações culinárias com significativa atividade antioxidante.

Todos os autores declararam não haver qualquer potencial conflito de interesses referente a este artigo.

\section{REFERÊNCIAS}

ALBERTI, A. Compostos fenólicos da maca: Extração, perfil e Classes Fenólicas, Atividade antioxidante, Processamento e Avaliação Termoanalítica. Curitiba, PR. Tese Doutorado. Universidade Federal do Paraná, 2014.

AMOROZO, M.C.M. Uso e diversidade de plantas medicinais em Santo Antônio do Leverger, MT, Brasil. Acta Bot. Bras. v.16, n.2, p.189-203, 2002.
$(22,45 \mathrm{mg} / \mathrm{mL})$ e sua respectiva farinha $(3,82 \mathrm{mg} / \mathrm{mL})$ pode observar que variou significativamente, mostrando que a farinha da polpa da maçã teve uma maior eficiência na capacidade de extrair os antioxidantes pelo método de DPPH. Segundo Balasundram et al. (2006), a atividade antioxidante encontrada nas farinhas avaliadas, pode servir como aditivos naturais em alimentos, resultando em dado importante, pois os antioxidantes sintéticos que são utilizados pela indústria de alimentos como BHA (butil-hidroxianisol), o BHT (butil- hidroxitolueno) e o TBHO (terc-butilhidroquinona) preocupam quanto as suas doses de segurança e toxicidade.

ALVES, L.F. Produção de Fitoterápicos no Brasil: História, Problemas e Perspectivas. Revista Virtual de Química. v. 5, n. 3, p. 450-513, 2013.

BALASUNDRAM, N.; SUNDRAM, K.; SAMMAN, S. Phenolic compounds in plants and agrindustrial byproducts: Antioxidant activity, occurrence, and potential uses. Food Chemistry, v.99, n.1, p.191-203, 2006.

BRAND-WILLIAMS， W.; CUVELIER, M.E.; BERSET, C. Use of a free radical method to evaluate antioxidant activity. Lebensmittel-Wissenschaft undTechnologie, London, v.28, n.1, p.25-30, 1995.

Ferreira, D.F. SISVAR - Sistema de análise de variância. Versão 5.3. Lavras-MG: UFLA, 2010.

ITO, V. Efeitos da Radiação gama durante o armazenamento da farinha do bagaço da maçã. Ponta Grossa, PR. Dissertação de Mestrado. Universidade Estadual de Ponta Grossa,2015.

NERIS, T. S.; SILVA S. S.; LOSS R. A.; CARVALHO J. P.; GUEDES S. F. Avaliação físicoquímica da casca da banana (musa spp.) in natura e desidratada em diferentes estádios de maturação. Ciência e Sustentabilidade - Ces, v.4, n.1, p.5-21, 2018.

QUEJI, M. D.; WOSIACKI, G.; CORDEIRO G. A.; PERALTA-ZAMORA, P. G.; NAGATA N. Determination of simple sugars, malic acid and total phenolic compounds in apple pomace by infrared 
spectroscopy and PLSR. International Journal of Food Science \& Technology, v.45, n.3, p.602-609, 2010.

SANTOS, I. D. Patulina em maçãs armazenadas em atmosfera controlada dinâmica utilizando UPLCMS/MS e monitoramento em supermercados. Santa Maria, RS. Dissertação de Mestrado. Universidade Federal de Santa Maria, 2017.

SHAHIDI, F. Nutraceuticals, functional foods and dietary supplements in health and disease. Journal of Food and Drug Analysis, v.20, n.1, p.226-230, 2012.
SOARES, M.; WELTER L.; KUSKOSKI E.M.; GONZAGA L.; FETT R. Compostos fenólicos e atividade antioxidante da casca de uvas Niágara e isabel. Revista Brasileira Fruticultura, v.30, n.1, p.59-64, 2008.

TSAU, R. ET AL. Which poliphenol compounds contribute to the total antioxidant activities of apple. Journal of Agricultural and Food Chemistry, v.53, n.12, p.4989-4995,2005.

WATERHOUSE, A. L. Polyphenolics: Determination of total phenolics. In R. E. Wrolstad (Ed.), Current protocols in food analytical chemistry. New York: John Wiley \& Sons, 2002. 